\title{
Management of OCDMA Auto-correlation Width by Chirp Manipulation Using SOA
}

\author{
Md Shakil Ahmed and Ivan Glesk, Senior Member, IEEE
}

\begin{abstract}
In order to preserve the performance of incoherent OCDMA systems it is imperative to maintain the width of the OCDMA auto-correlation function. This can be challenging in OCDMA systems when using multi-wavelength pico-second pulses as code carriers. In this respect, a Semiconductor Optical Amplifier (SOA) was used to control the chirp of OCDMA code carriers before fiber transmission, and the resulting effect on the recovered OCDMA auto-correlation was investigated at the receiving end after a $16 \mathrm{~km}$ long transmission in a fiber optic testbed with imperfectly balanced chromatic dispersion (CD).
\end{abstract}

Index Terms-Optical Code Division Multiple Access, OCDMA Auto-correlation, Chirp, Semiconductor Optical Amplifier, Chromatic Dispersion

\section{INTRODUCTION}

$\mathrm{T}$ HE deployment of incoherent OCDMA systems that use pico-second pulses as code carriers requires a chromatic dispersion (CD) compensated transmission link. If the link is not properly $\mathrm{CD}$ compensated, then the distortion due to pulse broadening and time-skewing would cause OCDMA autocorrelation function distortion at the receiving end. This would affect optical communication at chip level, cause inter chip interference resulting in bit-error-rate (BER) degradation [13]. It is therefore important to ensure that any deviations from the link's CD compensation are under control preferably via tunable means.

One approach to achieve tunable CD control is to exploit the signal's chirp before its transmission in the optical fiber. In [4], a laser pulse with a positive chirp was passed through SMF-28 fiber producing a negative chirp (due to fiber anomalous dispersion) leading to pulse compression. At the same time, due to group velocity dispersion (GVD) pulses have experienced broadening while propagating in optical fiber. At a distance known as the dispersion length $\left(L_{\mathrm{D}}\right)$, the pulse-width becomes equal to its initial value, seen at the fiber input due to the action of both of these simultaneous effects. After passing a distance $L_{\mathrm{D}}$, the propagated pulse will start to expand again until reaching the end of the fiber [9]. It is

Manuscript received ; revised ; accepted . Date of publication . This work was supported by the European Union's Horizon 2020 Research and Innovation Program under the Marie Skłodowska-Curie under Grant 734331.

M. S. Ahmed and I. Glesk are with the Electronic and Electrical Engineering Department, University of Strathclyde, Glasgow G1 1XW, U.K. (e-mail: shakil.ahmed@ strath.ac.uk; ivan.glesk@ strath.ac.uk).

Color versions of one or more of the figures in this letter are available online at http://ieeexplore.ieee.org.

Digital Object Identifier known that, either the initial positive chirp of a laser pulse or the resulting negative chirp due to fiber anomalous dispersion can be adjusted further to compress the optical pulse. For example, the initial positive chirp of the generated laser pulse can be reduced, or the length of SMF-28 fiber can be changed to alter it through its propagation in an optical fiber with anomalous dispersion. It has been shown that a Semiconductor Optical Amplifier (SOA) can also change the chirp of the optical laser pulse passing through the SOA by changing its bias current [5]. In [6] the effect of temperature-induced fiber dispersion on the OCDMA auto-correlation was mitigated by changing the gain of an SOA placed on the receiver side of the link. Through changes of the SOA gain dynamics and by introducing an optical holding beam, (cw and/or optical pulse synchronized with OCDMA auto-correlation at SOA input), the effect of fiber chromatic dispersion was investigated [7]. These control mechanisms affect the refractive index inside the SOA via gain changes (as predicted by Kramers-Kronig relations) resulting in a varied amount of chirp being imposed by the SOA on optical pulses passing through the SOA.

In this paper, we report for the first time the use of an SOA placed on the transmitter side to compensate OCDMA autocorrelation broadening on the receiver side caused by fiber chromatic dispersion. This is achieved by controlling the chirp of multi-wavelength OCDMA code carriers before the code is launched into a $16 \mathrm{~km}$ testbed. A developed equation allows theoretical and experimental investigation of chirp changes imposed by the SOA on the OCDMA auto-correlation. Our simulations are in a good agreement with experimental results.

\section{EXPERIMENT}

Figure 1 represents the experimental setup we used for our investigations. The OCDMA transmitter Tx produces a $2 \mathrm{D}$ WH/TS OCDMA code based on four wavelengths

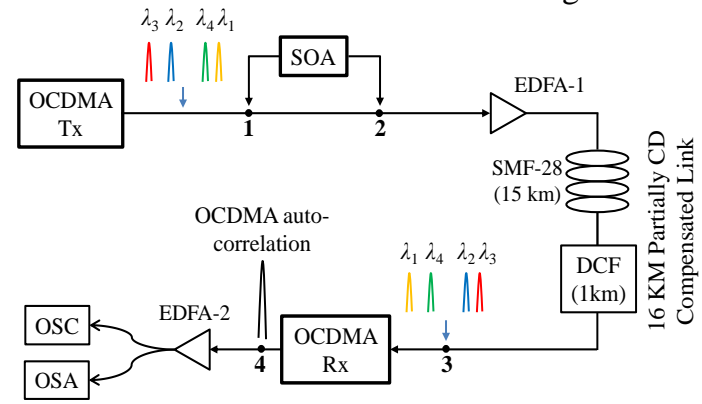

Fig. 1. Experimental setup. Tx - OCDMA transmitter, SOA - Semiconductor Optical Amplifier, EDFA - Erbium Doped Fiber Amplifier, DCF - Dispersion Compensating Fiber, SMF - Single Mode Fiber, Rx - OCDMA receiver, OSC - oscilloscope, OSA - Optical Spectrum Analyzer. 
$\left(\lambda_{1}=1551.72 \mathrm{~nm}, \lambda_{2}=1550.92 \mathrm{~nm}, \lambda_{3}=1552.52 \mathrm{~nm}, \lambda_{4}=\right.$ $1550.12 \mathrm{~nm}$ ) with 10 ps FWHM pulses as the code carriers. All pulse carriers have a positive chirp resulted from spectral slicing of the optical supercontinuum by a Fiber Bragg Gratings (FBGs) based 2D-WH/TS OCDMA encoder. A 2DWH/TS prime code is a class of two dimensional (2D: wavelength-time), incoherent asynchronous codes that support wavelength-hopping within time-spreading over the Galois field of prime numbers with zero auto-correlation side-lobes and periodic cross-correlation functions of at most one [8]. This transmitted code (2D- WH/TS OCDMA code, 1- $\lambda_{2}, 21$ $\lambda_{4}, 24-\lambda_{1}, 39-\lambda_{3}$ ) then propagates through a $16 \mathrm{~km}$ long CD compensated testbed connecting the University of Strathclyde and Glasgow University. A related OCDMA auto-correlation signal is then recovered by an OCDMA decoder ( $\mathrm{Rx})$ matched to the OCDMA encoder (Tx) at the fiber-receiving end (point4). For signal amplification, the EDFA-2 amplifier is used and the output is analyzed using an Optical Spectrum Analyzer (OSA - Agilent 86146B) and a Digitizing Oscilloscope, OSC (Agilent Infiniium DCA-J 86100C with $64 \mathrm{GHz}$ optical head).

The transmission link (Fig. 1) was first CD compensated by using the commercially available DCF module selected to match the $15 \mathrm{~km}$ SMF-28 fiber link. After its installation, we found there was a small chromatic dispersion mismatch and the link was narrowly over-compensated. That means the overall link is in the normal dispersion region $\left(\beta_{2}>0\right)$ having its equivalent link GVD parameter $\beta_{2}=0.2 \mathrm{ps}^{2} / \mathrm{km}$. To achieve tunability in $\mathrm{CD}$ compensation, and to make the link fully compensated without further adjustment of the DCF length, we investigated the use of SOA to fine-tune the compensation.

First, by performing 'back-to-back' measurements we have obtained the OCDMA auto-correlation temporal width in the absence of SOA at the Tx side (point-1). Then the autocorrelation was obtained after a $16 \mathrm{~km}$ propagation at the $\mathrm{Rx}$ receiver side (point-4). The results measured using the digitizing oscilloscope (OSC) are shown in Fig. 2(a) and 2(b),

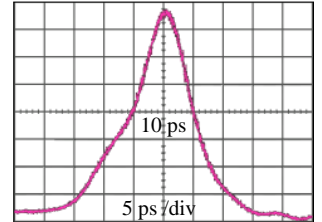

(a)

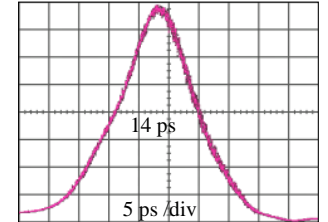

(b)
Fig. 2. Measured recovered OCDMA auto-correlation: a) back-to-back at point-1; b) After $16 \mathrm{~km}$ long fiber transmission at point- 4 .

respectively. From Fig. 2(b), we can see that, as the multiwavelength pico-second OCDMA pulses (code carriers) propagate through this $16 \mathrm{~km}$ long fiber testbed, the FWHM width of the recovered OCDMA auto-correlation function by the decoder Rx (point-4) has broadened from 10 ps to 14 ps when compared to the 'back-to-back' measurement shown in Fig. 2(a).

For $\beta_{2}=0.2 \mathrm{ps}^{2} / \mathrm{km}$, we can calculate the chromatic dispersion, $D_{\mathrm{CD}}[9]$ as:

$$
D_{\mathrm{CD}}=-\left(2 \pi c / \lambda^{2}\right) \times \beta_{2}
$$

Here, $c$ is the velocity of light in a vacuum. From Eq. (1), we have obtained a value of $D_{\mathrm{CD}}=-0.157 \mathrm{ps} /(\mathrm{nm} \bullet \mathrm{km})$ for the $\lambda=1550 \mathrm{~nm}$ region.

Now, to investigate the possibility of adjusting the FWHM temporal width of the recovered OCDMA auto-correlation function at the receiver end via controlling chirp of code carriers at the transmitting end by using SOA, an SOA (Kamelian OPA-20-N-C) was inserted between point 1 and 2 (see Fig. 1).

\section{THEORETICAL BACKGROUND AND ANALYSIS}

\section{A. Optical Pulse Chirp}

A pulse is chirped if its carrier frequency changes with time. The frequency change relates to the phase derivative. Parameter $C$ can be introduced to describe the linear frequency chirp imposed on the pulse. It depends on whether the instantaneous frequency of optical pulse is increasing or decreasing with time [5]. In the normal dispersion region, the GVD parameter $\beta_{2}$ is positive and the chirp is also positive, $C$ $>0$. At this condition, the higher frequency components' speed towards the pulse's leading edge is lower than the lower frequency components' speed at the trailing edge. On the other hand, in the anomalous dispersion region, the GVD parameter $\beta_{2}$ is negative and the chirp is also negative, $C<0$. The higher frequency components' speed towards the pulse's leading edge is faster than the lower frequency components' speed at the trailing edge. If the optical pulses generated by Tx (at point-1) have a positive chirp then after propagation through a normal dispersion region $\left(\beta_{2}>0\right)$, the positive chirp will become more positive (i.e., higher frequency components speed towards the leading edge will be even slower) and as a result, the pulse will be broadened. On the other hand, if these optical pulses will pass through a region with an anomalous dispersion $\left(\beta_{2}<0\right)$, then the positive chirp can be brought to zero. This will happen after traveling a distance known as $L_{\mathrm{D}}$. This leads to the compensation of GVD effect. Further propagation in the fiber (when distance $L$ exceeds length $L_{D}$ ) will lead to the pulse broadening as the effect of GVD becomes positive [10]. To summarize, an initial pulse narrowing happens if the product $C \beta_{2}<0$, and the travel distance $L<L_{\mathrm{D}}$, then if $L>L_{\mathrm{D}}$, the pulse broadens (until the end of the fiber link). The scenario described will also occur for optical pulses at the Tx output having a negative value of $C(C<0)$ where they would propagate in the optical fiber with normal dispersion $\left(\beta_{2}>0\right.$ and $\left.D_{\mathrm{CD}}<0\right)$ also leading to $C \beta_{2}<0$.

\section{B. Effect of Code Carriers Chirp on OCDMA Auto- correlation Temporal Envelope and Its Width}

A temporal Gaussian shape of an optical pulse affected by chirp $C$ is represented as [9]:

$$
P_{\mathrm{L}}(t)=P_{0} \exp \left[-2.77(1+i C)\left(\frac{t}{\tau}\right)^{2}\right]
$$

where $t$ is time, $P_{0}$ is a peak power and coefficient 2.77 indicates a pulse width $\tau$ measured at FWHM.

In our investigation, we have used four multi-wavelength pico-second pulses as 2D-WH/TS OCDMA code carriers, each of line-width $\Delta \lambda_{\mathrm{j}}$ and spectrally separated by $\Delta \Lambda_{\mathrm{j}}$ where $\mathrm{j}=1,2,3,4$, respectively. In light of Eq. (2), by following [11], for the OCDMA auto-correlation temporal envelope recovered 
by the Rx receiver (Fig. 1 point-4) we can write an expression:

$$
S_{\mathrm{L}\left(\lambda 1-\lambda^{4}\right)}(t)=\sum_{k=0}^{w-1} P \exp \left[-2.77(1+i C)\left(\frac{t-k \Delta \tau_{0}}{\tau-\Delta \tau_{0}}\right)^{2}\right]
$$

In the above expression, $w=4$ is a number of code carriers (wavelengths) also known as the code weight. The numerator $\left(t-k \Delta \tau_{0}\right)$ represents time skewing among wavelength carriers, the denominator $\left(\tau-\Delta \tau_{0}\right)$ reflects their spectral broadening due to dispersion effects and

$$
\Delta \tau_{0}=D_{\mathrm{CD}} \times L \times \Delta \lambda_{\mathrm{j}}
$$

where $D_{\mathrm{CD}}=-0.157 \mathrm{ps} /(\mathrm{nm} \bullet \mathrm{km})$ represents the dispersion of our partially compensated fiber testbed, $L=16 \mathrm{~km}$ is the testbed length, and $\Delta \lambda_{\mathrm{j}}=\Delta \Lambda_{\mathrm{j}}=0.8 \mathrm{~nm}$. The initial measured temporal FWHM value of wavelength code carriers is $\tau=8 \mathrm{ps}$.

By following [9], from Fig. 3, one can obtain the value of chirp $C$ affecting the resulting OCDMA auto-correlation $S_{\mathrm{L}}$ as: $C=\left[\left(\Delta \Omega \times a_{0}\right)^{2}-1\right]^{1 / 2}=\left[\left(\Delta \Omega \times \frac{\tau}{1.665}\right)^{2}-1\right]^{1 / 2}=+2.36$

Here $\Delta \Omega=3.42 \mathrm{~nm}$ (see Fig. 3) is the spectral width of the OCDMA auto-correlation found from the measurements at

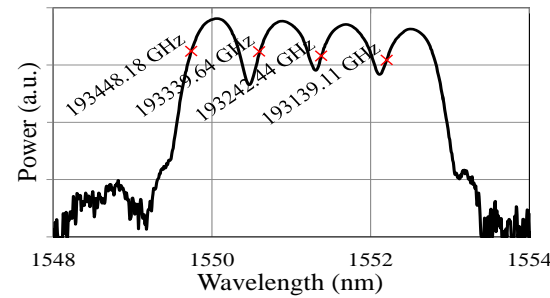

Fig. 3. Spectrum recorded during Back-to-Back measurements at point-1.

point-1 by the OSA (Agilent 86146B), and $a_{0}=\tau / 1.665$ reflects the use of the 'back-to-back' FWHM value of $\tau=10$ ps (see Fig. 2(a)).

The frequency domain representation of the OCDMA autocorrelation envelope after $L=16 \mathrm{~km}$ of propagation in the optical fiber can be obtained from Eq. (3) as follows [12]:

$S_{\mathrm{L}(\lambda 1-\lambda 4)}(f)=\mathrm{fft}\left\{S_{\mathrm{L}(\lambda 1-\lambda 4)}(t)\right\} \times\left\{\exp \left(-i \omega_{\mathrm{j}}^{2} \times \beta_{2} / 2\right) \times L\right\}(6)$

where $\beta_{2}=0.2 \mathrm{ps}^{2} / \mathrm{km}$ is the fiber link group velocity dispersion coefficient; $\omega_{\mathrm{j}}$ is an angular frequency of a particular code carrier $j$; and $\left\{\exp \left(-i \omega_{j}^{2} \times \beta_{2} / 2\right) \times L\right\}$ factors in the GVD effects imposed by propagation in the fiber.

In order to obtain the OCDMA auto-correlation function at the fiber end in the time domain, we perform an inverse Fourier transform on Eq. (6):

$$
S_{\mathrm{L}(\lambda 1-\lambda 4)}(\mathrm{t})=\text { ifft }\left\{S_{\mathrm{L}(\lambda 1-\lambda 4)}(f)\right\}
$$

Now, using $C=+2.36$ found from Eq. (5) and substituting Eq. (3) and (6) into Eq. (7), it is possible to calculate the 2DWH/TS OCDMA auto-correlation envelope $S_{\mathrm{L}}$ recovered by the matched OCDMA receiver $\mathrm{Rx}$ at point- 4 after $L=16 \mathrm{~km}$ long transmission (Fig. 4(b)) and the related FWHM width is 14.2 ps. The corresponding experimental measurement shows the FWHM value of 14 ps (Fig. 4(a)). For a comparison, the 'back-to-back' measured and simulated FWHM widths for the OCDMA envelope are also shown in Fig. 4(a) and 4(b), respectively each having the same value of $10 \mathrm{ps}$.

Based on [10] we can see that, the larger $C>0$ code carriers have at Tx output, the more broadening they will experience during their fiber propagation. However, if the $C$ value can be reduced, the OCDMA auto-correlation widths observed at the fiber output will be also reduced. This will be explored next.

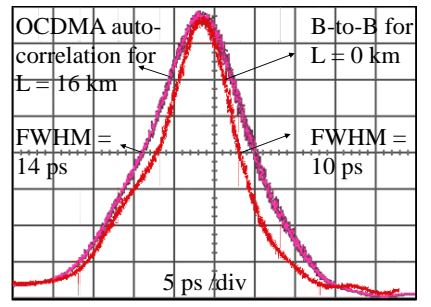

(a) Experiment

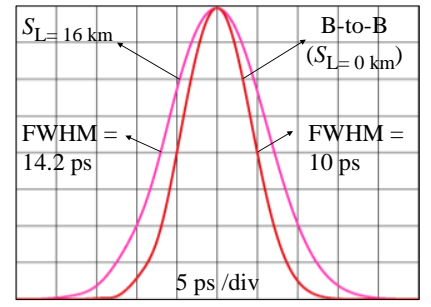

(b) Simulations
Fig. 4(a). Measured 2D-WH/TS OCDMA auto-correlation recovered by a matched OCDMA decoder Rx for back-to-back $(L=0)$ and after $L=16 \mathrm{~km}$ propagation; (b) Simulations for the same.

\section{Chirp variation by SOA}

To explore the possibility of reducing the $C$ value, we have added an SOA after the Tx in between points 1 and 2 (see Fig. 1). This allows us to vary the SOA's gain dynamics through a change in bias current $I$ (carrier density), which results in refractive index changes thus causing a phase shift $[9,10]$. Before going any further we took the optical spectrum of 2DWH/TS code immediately after passing the SOA biased at $I=$ $7 \mathrm{~mA}$. The measured result is shown in Fig. 5. Now recall, Fig. 3 shows the code optical spectrum at the SOA input. By

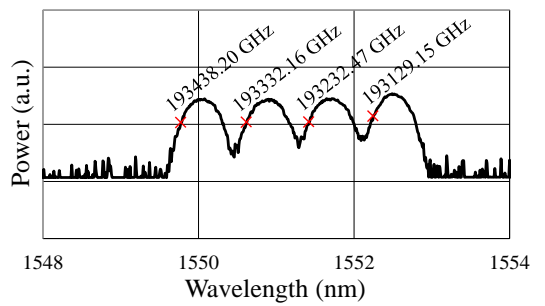

Fig. 5. Spectrum recorded at SOA output (point-2) at bias current $I=7 \mathrm{~mA}$.

comparing it with Fig. 5 we obtained the respective spectral frequency shifts $\Delta v\left(\lambda_{4}\right)=9.98 \mathrm{GHz}$ for $\lambda_{4}, \Delta v\left(\lambda_{2}\right)=7.48 \mathrm{GHz}$ for $\lambda_{2}, \Delta v\left(\lambda_{1}\right)=9.97 \mathrm{GHz}$ for $\lambda_{1}$ and $\Delta v\left(\lambda_{3}\right)=9.96 \mathrm{GHz}$ for $\lambda_{3}$ imposed by the SOA biased at $I=7 \mathrm{~mA}$ resulting in a frequency chirp of $\Delta v=9.34 \mathrm{GHz}$. In order to find the related chirp parameter $C_{\text {out }}$ induced by SOA, we have used [13]:

$$
C_{\text {out }}=\Delta v(\mathrm{GHz}) \times 2 \pi \sigma=0.25
$$

where $\sigma=\tau / 2.35$ and $\tau=10$ ps are the measured FWHM value of code carriers entering the SOA. The coefficient 2.35 is a result of conversion between the FWHM width $\tau$ of a Gaussian shape pulse and standard deviation $\sigma$. Now, after substituting $C=C$ out $=0.25$ into Eq. (3) and following steps as before, from Eq. (7) it is possible to calculate the 2D-WH/TS OCDMA auto-correlation envelope $S_{\mathrm{L}}$ recovered by the OCDMA decoder $\mathrm{Rx}$ after an $L=16 \mathrm{~km}$ long fiber transmission at point-4 and the related FWHM width value. The result of this simulation is shown in Fig. 6(b) as curve $C=$ 0.25 . Its calculated FWHM width is $10.6 \mathrm{ps}$. In contrast, the curve marked $I=7 \mathrm{~mA}$ in Fig. 6(a) is the experimentally recovered OCDMA auto-correlation at point-4 for SOA current $I=7 \mathrm{~mA}$. The corresponding FWHM value is $11 \mathrm{ps}$. Results of our measurements for different $I$ values are also included in Fig. 6(a) together with results of OCDMA autocorrelation envelope simulations for different chirp values $C$ in Fig. 6(b). Then OCDMA auto-correlation FWHM values as a function of SOA current $I$ were extracted from Fig. 6(a). 


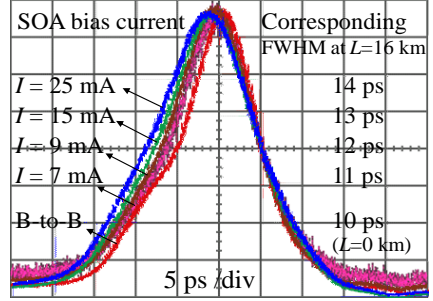

(a) Experiment

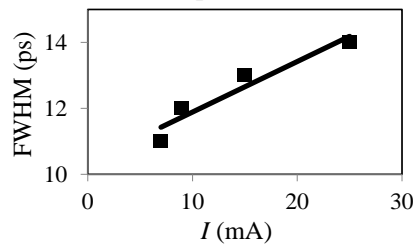

(c) Experiment

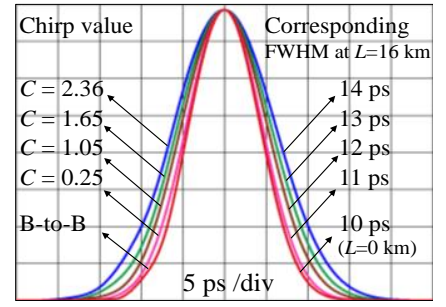

(b) Simulations

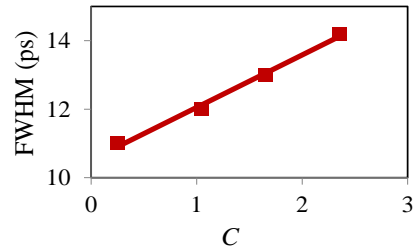

(d) Simulations
Fig. 6(a). Measured 2D-WH/TS OCDMA auto-correlation envelope for different SOA bias currents $I$ after $L=16 \mathrm{~km}$ fiber propagation; (b) Simulated 2D-WH/TS OCDMA auto-correlation envelope after $16 \mathrm{~km}$ fiber propagation for different values of chirp $C$; (c) OCDMA auto-correlation FWHM width vs SOA bias current $I$; (d) OCDMA auto-correlation FWHM width vs chirp $C$.

These results are shown in Fig. 6(c). Similarly, the OCDMA auto-correlation FWHM values as a function of chirp $C$ were extracted from Fig. 6(b) and results were plotted in Fig. 6(d). Looking at the simulation results, we can see that for a $16 \mathrm{~km}$ long propagation in the fiber testbed, $C=0.25$ delivered the max. auto-correlation width reduction, from 14 ps to $10.6 \mathrm{ps}$. This was then confirmed experimentally where for the SOA bias current $I=7 \mathrm{~mA}$, the FWHM value $11 \mathrm{ps}$ is also minimal. Any further decreases of $I$ in our attempts to achieve a 'backto-back' $\tau=10$ ps value of the OCDMA auto-correlation width also for $L=16 \mathrm{~km}$ led to an impractically low SOA gain. To overcome this, we used a continuous wave (CW) holding beam technique (CW/HB) [7]. The $\mathrm{CW}$ source (see Fig. 7(a))

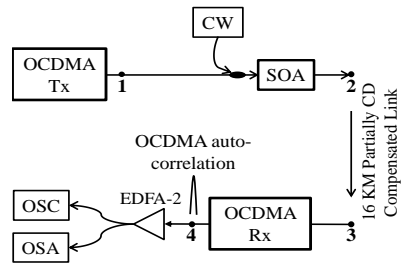

(a)

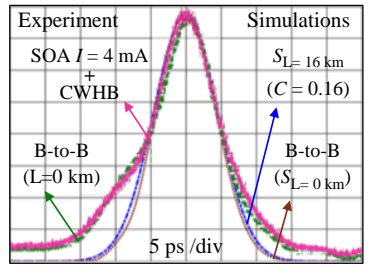

(b)
Fig. 7(a). Testbed setup after adding CW source as CWHB. (b) Corrected OCDMA auto-correlation envelope: red line - measurement after $L=16 \mathrm{~km}$ propagation (point-4) for the SOA bias current $I=4 \mathrm{~mA}$ in the presence of CW and blue dot-dashed line - simulation when SOA induced chirp parameter is $C=0.16$. Brown dotted (green dashed) line is the $S_{\mathrm{L}=0 \mathrm{~km}}$ simulation (backto-back measurement at point-1), respectively.

was an SOA (Kamelian OPA-20-N-C) with its gain set to one. A collinearly added $\mathrm{CW}$ enhances the stimulated emission in the SOA [14], and thus the SOA gain dynamics. In this configuration and with an SOA bias current of $I=4 \mathrm{~mA}$ we were able to experimentally achieve the OCDMA FWHM value of 10 ps (see Fig. 7(b) red line) which is in an agreement with 'back-to-back' measurements (green dashed line).

Now, using the steps described earlier, we found the resulting value of the chirp at point-2 as $C=0.16$. By using Eq. (7), we found the 'corrected' OCDMA auto-correlation envelope $S_{\mathrm{L}=16}$ $\mathrm{km}$ as shown in Fig. 7(b) by the blue dot-dashed line. It can be seen that its OCDMA FWHM value is also $10 \mathrm{ps}$ and matches well with our back-to-back measurements (green dashed line) and the related simulations $S_{\mathrm{L}=0 \mathrm{~km}}$ (brown dotted line).

\section{CONCLUSION}

We have shown for the first time both experimentally and by simulation, that a Semiconductor Optical Amplifier at the transmission side can be used to control a recovered 2DWH/TS OCDMA auto-correlation width at the receiver end by controlling the chirp of pico-second multi-wavelength OCDMA code carriers before the code transmission. Our experiments and simulations were conducted for a $16 \mathrm{~km}$ long fiber transmission in a partially CD compensated fiber testbed connecting Strathclyde and Glasgow Universities. We have found that OCDMA auto-correlation and its $14 \mathrm{ps}$ width when $\mathrm{CD}$ distorted by a fiber transmission can be restored back to its 10 ps 'back-to-back' value by controlling the code carriers' chirp by varying the SOA bias current. Using $\mathrm{CW} / \mathrm{HB}$ does not offer the same CD tuning range as OP/HB [7], however using $\mathrm{CW} / \mathrm{HB}$ eliminates the need for synchronization and simplifies system implementation. Because the SOA faces code carriers having low power and equal amplitude, no bandwidth limitations were noted which is contrary to [7] where the SOA is implemented on the receiving end.

\section{REFERENCES}

[1] E. K. Ng, G. E. Weichenberg, and E. H. Sargent, "Dispersion in multiwavelength optical code-division multiple-access systems: impact and remedies," IEEE Trans. Commun., vol. 50, no.11, pp. 1811-1816, Nov. 2002.

[2] A. Sahin and A. E. Willner, "System limitations due to chromatic dispersion and receiver bandwidth for 2-D time-wavelength OCDMA systems," in Proc. 16th Annual Meeting of the IEEE, vol. 2. IEEE, 2003.

[3] S. P. Majumder, A. Azhari, and F. M. Abbou, "Impact of fiber chromatic dispersion on the BER performance of an optical CDMA IM/DD transmission system," IEEE Photon. Technol. Lett., vol. 17, no. 6, pp. 1340-1342, Jun. 2005.

[4] S. T. Cundiff, B. C. Collings, L. Boivin, M. C. Nuss, K. Bergman, W. H. Knox, and S. G. Evangelides, "Propagation of highly chirped pulses in fiber-optic communications systems". J. Lightwave Technol., vol. 17, no. 5, pp. 811-816, May. 1999.

[5] M. N. Ngo, H. T. Nguyen, C. Gosset, D. Erasme, Q. Deniel, and N. Genay, "Transmission performance of chirp-controlled signal emitted by electroabsorption modulator laser integrated with a semiconductor optical amplifier," in Optical Fiber Communication Conference, Optical Society of America, pp. OW4F-6, March. 2012.

[6] M. S. Ahmed and I. Glesk, "Mitigation of temperature induced dispersion in optical fiber on OCDMA auto-correlation," IEEE Photon. Technol. Lett., vol. 29, no 22, pp. 1979-1982, Nov. 2017.

[7] M. S. Ahmed, M. S. K. Abuhelala, and I. Glesk, "Managing dispersionaffected OCDMA auto-correlation based on PS multiwavelength code carriers using SOA." IEEE/OSA J. Opt. Commun. Net., vol. 9, no. 8, pp. 693-698, Aug. 2017.

[8] G. C. Yang and W. C. Kwong, Prime Codes with Applications to CDMA Optical and Wireless Networks. Artech House, Boston, 2002.

[9] G. P. Agrawal. Nonlinear fiber optics. Academic press, 2007.

[10] H. Abramczyk, "Dispersion phenomena in optical fibers," Virtual European University on Lasers. [Online]. Available: http://www.mitr.p.lodz.pl/evu/wyklady/.

[11] T. B. Osadola, S. K. Idris, I. Glesk, and W. C. Kwong, "Effect of variations in environmental temperature on 2D-WH/TS OCDMA code performance," J. Opt. Commun. Net., vol. 5, no. 1, pp. 68-73, Jan. 2013.

[12] E. M. Wright, "Module 3-Numerical Pulse Propagation in Fibers." [Online]. Available: http://data.cian-erc.org/supercourse/Graduatelevel/ module_3/3_SC_GRAD_LEVEL_Module3_wright.pdf.

[13] G. P. Agrawal and M. J. Potasek. "Effect of frequency chirping on the performance of optical communication systems." Optics Lett., vol. 11, no. 5, pp. 318-320, May 1986.

[14] H. N. Tan, M. Matsuura, and N. Kishi, "Enhancement of input power dynamic range for multiwavelength amplification and optical signal processing in a semiconductor optical amplifier using holding beam effect," J. Lightwave Technol., vol. 28, no. 17, pp. 2593-2602, 2010. 